INPLASY

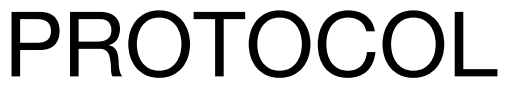

To cite: Ma et al. Efficacy and safety of adult stem cell transplantation for T1MD patients and animals: a metaanalysis. Inplasy protocol 202190090. doi:

10.37766/inplasy2021.9.0090

Received: 25 September 2021

Published: 25 September 2021

Corresponding author:

Yijin Pei

pyj@gdmu.edu.cn

Author Affiliation:

Guangdong Medical University

Support: National Natural

Science Found.

Review Stage at time of this submission: Piloting of the study selection process.

Conflicts of interest:

None declared.

\section{Efficacy and safety of adult stem cell transplantation for T1MD patients and animals: a meta-analysis}

Ma, HF'; Qiu, JX2; Mai, PJ3; Pei, YJ4.

Review question / Objective: The aim of this meta-analysis is to evaluate the efficacy and safety of adult stem cell transplantation for type 1 diabetes.

Condition being studied: Type 1 diabetes is a metabolic disorder syndrome characterized by high blood sugar due to an absolute lack of insulin. The disease is mainly caused by immune-mediated destruction of beta cells in the pancreas. Due to the destruction of beta cells in the pancreas, insulin secretion is relatively insufficient, and multiple organs of the body are exposed to hyperglycemia for a long time. In recent years, the incidence of T1DM has been increasing year by year ( $3 \% \sim 5 \%$ per year), young T1DM patients ( $<20$ years old) more than 1 million, and if current trends continue, it is expected to grow by 100,000 a year. T1MD is currently treated with insulin, but insulin is prone to cause adverse reactions such as hypoglycemia and increase in body weight, and patients' compliance is low.Stem cells are versatile and can differentiate into various cells in vivo or in vitro, so they can be transplanted into the body to replace the function of islet $\beta$ cells and lower blood sugar.

INPLASY registration number: This protocol was registered with the International Platform of Registered Systematic Review and Meta-Analysis Protocols (INPLASY) on 25 September 2021 and was last updated on 26 September 2021 (registration number INPLASY202190090).

\section{INTRODUCTION}

Review question / Objective: The aim of this meta-analysis is to evaluate the efficacy and safety of adult stem cell transplantation for type 1 diabetes.
Condition being studied: Type 1 diabetes is a metabolic disorder syndrome characterized by high blood sugar due to an absolute lack of insulin. The disease is mainly caused by immune-mediated destruction of beta cells in the pancreas. Due to the destruction of beta cells in the 
pancreas, insulin secretion is relatively insufficient, and multiple organs of the body are exposed to hyperglycemia for a long time. In recent years, the incidence of T1DM has been increasing year by year (3\% 5\% per year), young T1DM patients (< 20 years old) more than 1 million, and if current trends continue, it is expected to grow by 100,000 a year. T1MD is currently treated with insulin, but insulin is prone to cause adverse reactions such as hypoglycemia and increase in body weight, and patients' compliance is low.Stem cells are versatile and can differentiate into various cells in vivo or in vitro, so they can be transplanted into the body to replace the function of islet $\beta$ cells and lower blood sugar.

\section{METHODS}

Search strategy: We will search articles in four electronic database including NCBI, Cochrane Library, EMBASE, and MEDLINE. All the English publication until 30 June 2021 will be searched without any restriction of countries and article types.We used a combination of MeSH term and free words: ('Stem Cell Transplantation'[MeSH] OR 'Transplantations, Stem Cell' OR 'Transplantation, Stem Cell') AND ('Diabetes Mellitus, Type 1'[Mesh] OR 'Diabetes Mellitus, Insulin-Dependent' OR 'Insulin-Dependent Diabetes Mellitus' OR 'IDDM' OR 'Type 1 Diabetes Mellitus' OR 'Type 1 Diabetes' OR 'Diabetes Mellitus, Juvenile Onset').

Participant or population: Participants who were diagnosed with type 1 diabetes will be included.

Intervention: Stem cell transplantation was the main intervention.

Comparator: The same dose of insulin or a placebo was injected into the body.

Study designs to be included: Articles without any restriction of countries and types were included and all the English publication until 30 June 2021 will be searched.
Eligibility criteria: Non-case reports, reviews, reviews, conference summaries, in vitro studies, and study designs with no significant flaws, good quality, complete outcome data, and clear efficacy will be reported.

Information sources: We will search articles in three electronic database including NCBI, Cochrane Library, EMBASE, and MEDLINE. If the article is incomplete, the author will be contacted for data supplement.

Main outcome(s): HbA1C, C peptide and insulin dosage/production.

Additional outcome(s): body weight and blood sugar.

Data management: We will use Endnote X9 for data management.

Quality assessment / Risk of bias analysis: Two researchers used the Cochrane Collaboration's tool for randomized controlled trails and MINORS for NonRandomized Concurrent Controlled Trial to evaluate the quality of articles. Items will be evaluated in three categories: Low risk of bias, unclear bias and high risk of bias. The following characteristics will be evaluated:Random sequence generation (selection bias), Allocation concealment (selection bias),Blinding of participants and personnel (performance bias),Blinding of outcome assessment (detection bias), Incomplete outcome data (attrition bias),Selective reporting (reporting bias) and Other biases. Results from these questions will be graphed and assessed using Review Manager 5.4.

Strategy of data synthesis: Continuous variables were used as efficacy analysis statistics with Standard Mean Difference (SMD) or Weighted Mean Difference (WMD) and $95 \%$ confidence interval $(95 \% \mathrm{Cl})$. The counting data (such as the adverse reactions) were used as the statistics of efficacy analysis by related risk (RR) and $95 \% \mathrm{Cl}$. I ${ }^{2}$ value was used to test inter-study heterogeneity. If there was no significant clinical heterogeneity between studies 
$\left(I^{2}<50 \%, P<0.1\right)$, using the fixed effects model for meta-analysis; If $I^{2} \geq 50 \%$, clinical heterogeneity was indicated among all groups. The source of heterogeneity was analyzed. Subgroup analysis and sensitivity analysis were performed if necessary. Funnel plots were used to analyze possible publication bias.

Subgroup analysis: We will consider subgroups such as $\mathrm{HbA1c}, \mathrm{CP}$ and insulin dosage.

Sensitivity analysis: Excluding low-quality studies, if there is no significant change in the results after exclusion, it indicates low sensitivity and reliable results. On the contrary, if large differences or even completely opposite conclusions are obtained after exclusion, it indicates high sensitivity and low robustness of the results.

Language: All the English publication until 30 June 2021 will be searched.

Country(ies) involved: China.

Keywords: Stem cell transplantation; Type 1 diabetes; Clinical trails; Animal experience.

Contributions of each author:

Author 1 - Huifen Ma - The author carried out subject determination, literature retrieval, data extraction, mapping and paper writing.

Email: mahuifen1998@163.com

Author 2 - Jiaxin Qiu - The author collated the data and provided statistical expertise.

Email: 527881992@qq.com

Author 3 - Peiju Mai - The author revised the paper.

Email: 964476021@qq.com

Author 4 - Yijin Pei - The author is the corresponding author. The author read, provided feedback and approved the final manuscript. The author suggests modifications.

Email: pyj@gdmu.edu.cn 